\title{
XIV.
}

Arbeiten aus dem pharmakologischen Institut der dentschen Universität zu Prag.

\section{Ueber Resorption und Assimilation der Nährstoffe.}

Fünfte Mitheilung.

Ueber die Assimilationsgrenze der Zuckerarten.

Von

Franz Hofmeister.

Die Lehre vom Diabetes mellitus ist so innig mit der Lehre von der Assimilation der Kohlenhydrate verkniipft, dass alle Untersuchungen, welche sich auf die eine beziehen, auch der anderen zu Gute kommen. Viele von den einschlägigen physiologischen Untersuchungen, auch der neuesten Zeit, lassen ihren Ursprung auf einen von der klinischen Beobachtung ausgehenden Anstoss zuriekführen. Es muss dahingestellt bleiben, ob sich die gegenseitige Beeinflussung von Theorie und Praxis in jedem einzelnen Falle als förderlich erwiesen hat. Genug daran, dass dieselbe, wenngleich oft auf längeren Umwegen, doch immer wieder zur Gewinnung neuer, sicher beobachteten Thatsachen Anlass gab.

$\mathrm{Zu}$ den einschlägigen Fragen, welche bisher vorwiegend von klinischen Gesichtspunkten aus, zum Theil allerdings gerade von Seiten der Physiologen Bearbeitung gefunden haben, zählt auch die im Nachstehenden behandelte, nach der Grenze der Assimilirbarkeit der Zuckerarten. Seit man begonnen hat, durch Fütterungsversuche uiber das Schicksal der Nährstoffe Aufschluss zu suchen, ist von den Beobachtern immer wieder angegeben worden, dass nach übermässiger Zufuhr von Zucker oder von Koblenhydraten schlechtweg Ausscheidung von Zncker mit dem Harne erfolgen kann. Diese Beobachtungen sind jedoch an sich sehon mit Vorsicht aufzunehmen, da in zahlreichen Fällen der Zuckernachweis nicht mit genügend zuverlässigen Methoden geführt erscheint. Der weitere Umstand, dass bei solchen Fütterungsversuchen verschiedene Kohlenhydrate, zum 
Theil sogar in ihrer Zusammensetzung unbekannte Nahrungsgemenge in Verwendung kamen, dass iiberdies die Versuche nicht an derselben Thierspecies angestellt wurden, macht es begreiflich, dass die einschlägigen Beobachtungen von Widersprüchen wimmeln, und erschwert die Gewinnung einfacherer Vorstellungen ausserordentlich.

So konnten Budge ${ }^{1}$ ) und Baumert ${ }^{2}$ ) beim Kaninchen nach reichlicher Zufuhr von Rohrzucker keine Glykosurie nachweisen, während J.M. S chiff ${ }^{3}$ ) nach Fütterung ron gezuckertem Mohrrübenbrei zu dem entgegengesetzten Schlusse kam.

v. Beeker ${ }^{4}$ ) fand bei vielen Versuchen nur einmal ein Kaninchen, welches bei Möhrendiät Zucker im Harn hatte. Von $30 \mathrm{Ka}$ ninchen, denen 0,220-0,962 $\mathrm{g}$ Traubenzucker in den Magen gespritzt worden war, lieferten nur 5 dentlich zuckerhaltigen Harn.

Beim Pferd soll Boussingault, einer Angabe bei Lecor$\operatorname{ch}^{5}$ ) zufolge, nach Fiutterung mit $12 \mathrm{~kg}$ Rohrzuèker keine Glykosurie haben folgen sehen.

Beim $\mathrm{Hund}$ sah F. Hoppe ${ }^{6}$ ) bei seinen ausgedehnten Futterungsversuchen mit Rohrzucker, trotz sehr reichlicher Zufuhr (bis $200 \mathrm{~g}$ ) nie auch nur eine Spur Zucker in den Harn übertreten, während $\mathrm{Budge}, \mathrm{Poggiale}$ und $\mathrm{Cl}$. Bernard uiber entgegengesetzte Beobachtungen berichten. Budge (a. a. O.) fand den Harn eines jungen weiblichen Hundes, welcher 4 Loth (etwa $70 \mathrm{~g}$ ) Rohrzucker erhalten hatte, reducirend. Poggiale ${ }^{7}$ konnte in einem Versuch, wo viel Zucker und Stärkemehl enthaltende Nabrung neben doppeltkohlensaurem Natron verfiittert worden war, in dem stark alkalischen Harn 5-7 g Glykose auf 1000 ecm nachweisen. Cl. Bernard ${ }^{8}$ ) erwähnt wiederholt, nach Darreichung grosser Dosen Rohrzucker (20-80 g pro Hund) den Harn stark reducirend und linksdrehend gefunden zu haben.

Bei der Katze hat C. Schmidt ${ }^{9}$ ) sowohl nach Einfuhrung von Rohrzuckerlösung in den Magen, als nach Fütterung von mit dem halben Gewicht Rohrzucker versetzter Milch reichlich reducirenden und gährungsfähigen Zucker im Harn gefunden.

1) Archiv für physiol. Heilkunde. III. Bd. S. 402. 1844.

2) Journ. für prakt. Chemie. LIV. Bd. S. 357. 1851.

3) Untersuchungen üb. die Zuckerbildung in d. Leber etc. Würzburg 1859. S. 45.

4) Zeitschrift für wissenschaftl. Zoologie V. Bd. S. 123.

5) Le Diabète. Paris 1877. p. 502.

6) Virchow's Archiv. 10. Bd. S. 144.

7) Compt. rend. Vol. XLiII. p. 198. 1856.

8) Leçons sur le diabète. Paris 1877. p. 270, 320.

9) Charakteristik der epidem. Cholera. Leipzig u. Mitau 1850. S. 165. 
Beim gesunden Menschen sind durch die sorgfältigen Untersuchungen Worm-Müller's ${ }^{1}$ ) die früheren Angaben, welche theils den Uebertritt von Zucker aus der Nahrung in den Harn behaupteten (Mosler, L. Vogel, Gubler u. A.), theils leugneten (Budge, C. G. Lehmann, Kersting, v. Frerichs u. A.), weit überholt worden.

Worm-Müller stellte seine Versuche an zwei gesunden Individuen an, welche während der Dauer des Versuches neben dem gereichten Kohlenhydrat nur zuckerfreie Nahrung erhielten. Von Zuckerarten kamen bei den Versuchen in Verwendung: Rohrzucker, Milchzucker, Traubenzucker und, in Ermangelung reinen Fruchtzuckers, Honig, dessen Gehalt an Trauben- und Fruchtzucker genau ermittelt worden war. Bei Untersuchung des Harns bestimmte er sorgfältig Art und Menge des übergetretenen Zuckers. Das Ergebniss seiner Versuche lässt sich dahin zusammenfassen, dass von Milch-, Robr- und Traubenzucker, wenn sie in grösserer Menge (zum mindesten $50 \mathrm{~g}$ auf einmal) gereicht werden, kleine Quantitäten innerhalb der ersten 3-5 Stunden in den Harn übergehen, während Levulose, in etwa gleich grosser Menge genossen, nicht im Harn aufzufinden ist. Dieses Ergebniss lässt sich in anderer Form so ausdrücken: Es giebt beim Menschen für die Zufuhr von Rohr-, Milch- und Traubenzucker (nicht aber für Levulose) eine Grenze, bei deren Ueberschreitung ein Theil des eingefuhrten Zuckers im Harn erscheint.

Die Menge des in den Versuchen Worm-Müller's von den Versuchspersonen ausgeschiedenen Zuckers ist aus nachstehender $\mathrm{Zu}$ sammenstellung ersichtlich:

\begin{tabular}{|c|c|c|}
\hline $\begin{array}{l}\text { Vesuchs- } \\
\text { person }\end{array}$ & Gewieht & Im Harn wieder gefunder \\
\hline T. T. & $250 \mathrm{~g}$ Rohrzucker & $1,81 \mathrm{~g}$ Rohrzucker \\
\hline V. C. & $150 \mathrm{~g}=$ & $0,85 \mathrm{~g}=$ \\
\hline V. $\mathbf{C}$ & $50 \mathrm{~g}=$ & ca. $0,1 \mathrm{~g}=$ \\
\hline V. C. & $200 \mathrm{~g}$ Milehzucker & $1,68 \mathrm{~g}$ Milchzucker \\
\hline V. C. & $100 \mathrm{~g}=$ & $0.32 \mathrm{~g}=$ \\
\hline V.C. & $\quad 50 \mathrm{~g}$ Traubenzucker & $0,47 \mathrm{~g}$ Traubenzucker \\
\hline T. T. & $117 \mathrm{~g}$ Traubenzucker und $86 \mathrm{~g}$ Fruehtzucker & $1,30 \mathrm{~g}=$ \\
\hline V.C. & $58 \mathrm{~g} \quad=\quad=42 \mathrm{~g}=$ & $0,81 \mathrm{~g}$ \\
\hline
\end{tabular}

Die Zahl dieser Versuche gestattet nicht, allgemeine Schliisse mit Sicherheit aus denselben zu ziehen. Immerhin geben sie mehr als einen werthvollen Fingerzeig für eine Weiterführung einschlägiger Untersuchungen. So fällt auf, dass bei derselben Versuchsperson nach Genuss von $50 \mathrm{~g}$ Rohzucker viel weniger Zucker im Harn erschien,

1) Pflüger's Archiv für die ges. Physiologie. XXXIV. Bd. S. 576. 
als nach Zufuhr der gleichen Menge Dextrose. Es deutet dies darauf hin, dass die Assimilationsgrenze für die verschiedenen Zuckerarten eine verschiedene ist. Ferner ist ersichtlich, dass die Menge des in den Harn übertretenden Zuckers um so grösser ausfällt, je mehr Zucker eingeführt wurde, dass aber diese Steigerung nicht etwa derart zu Stande kommt, dass über eine gewisse Grenze hinaus der gesammte überschüssig zugeführte Zucker im Harn zum Vorschein käme. In dieser Richtung ist namentlich ein Vergleich der drei Rohrzuckerversuche von Interesse.

Die eben angedeuteten Beziehungen zwischen Zuckerzufuhr und Zuckerausscheidung dürften, wie sich aus den einschlägigen Ver-

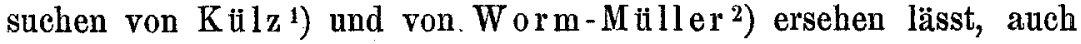
für den Zuckerverbrauch des Diabetikers Geltung beanspruchen. Der Diabetiker leichterer Form zeigt bei sonst streng gehandhabter Fleischkost eine gewisse "Toleranz" für Zucker und für Amylaceen, insofern er nur bei Zufuhr derselben über eine gewisse Grenze hinaus zuckerhaltigen Harn liefert. Er besitzt noch die Fähigkeit, einen grossen Theil der eingeführten Kohlenhydrate völlig zu verbrauchen. Auch dem Diabetiker der schwereren Form geht diese Fähigkeit nicht ganz ab. Scheidet er bei strenger Fleischnahrung täglich eine annähernd gleiche Zuckermenge aus, so hat die Einführung einer gewissen Menge von Kohlenhydraten nicht eine Steigerung des im Harn erscheinenden Zuckers um eine äquivalente, sondern nur um eine viel kleinere Quantität zur Folge. Soweit es sich dabei um Zufuhr von Zuckerarten handelt, besteht in dieser Richtung zwischen dem Gesunden und dem Diabetiker kein qualitativer, sondern nur ein quantitativer Unterschied.

Bei Anstellung der im Nachfolgenden mitgetheilten Versuche hatte ich im Ange, zu untersuchen, ob die oben angedeuteten $\mathrm{Be}-$ ziehungen zwischen Zufuhr und Ausscheidung von Zucker allgemeinere Gültigkeit, namentlich auch für das zu Fütterungsversuchen am besten geeignete Versuchsthier, den Hund, besitzen. Ich war dabei von der Hoffnung geleitet, durch Klarstellung dieser Beziehungen einen festen Boden zu gewinnen, von welchem aus auf dem Wege des Thierversuches die Frage nach dem Schicksal der in den Körper eingeführten Kohlenhydrate und nach der Entstehung des Diabetes neuerlich in Angriff genommen werden könnte.

Versuchsanordnung. $\mathrm{Zu}$ den Versuchen dienten ausschliesslich kleine weibliche Hunde. Sie wurden in kleinen Käfigen gehalten,

1) Beiträge zur Pathologie und Therapie des Diabetes. I. Bd. S. 98 ff.

2) Pflüger's Archiv. XXXVI: Bd. S. 172. 
deren geneigter und mit Abflussvorrichtung versehener Zinkboden Sammeln und Nachspülen des Harns ohne Verlust ermöglichte. Die Thiere nicht frei herumlaufen zu lassen, erschien schon im Hinblick auf die von $\mathrm{K} \ddot{u} \mathrm{lz}$ u. A. bei Diabetikern gemachte Erfabrung geboten, dass starke körperliche Bewegung auf die Grösse des Zuckerverbrauches Einfluss nimmt. Die benutzten Zuckerarten waren: Dextrose Galaktose, Levulose, Rohrzucker und Milchzucker.

Die Fitterung der Thiere geschah in der Art, dass dieselben zuerst den Zucker, in ziemlicher Concentration in Fleischsuppe gelöst in einer Schale vorgesetzt erhielten, sodann aus derselben Schale neuerlich eine Portion Fleischbriihe (mit oder ohne Zusatz einer gewogenen Menge Fleisch) verzebrten. Dies geschah in der Regel bis auf den letzten Tropfen, so dass ein Verlust an Zucker ausgeschlossen war. Die Menge der im Ganzen eingefuihrten Flïssigkeit wurde absichtlich ziemlich hoeh bemessen, siebetrug 200-350 ccm. Die Fütterung selbst wurde ausserhalb des Käfigs vorgenommen; auch wurde darauf geachtet, dass keine Verunreinigung des Käfigs durch an Maul oder Pfoten haftende zuckerhaltige Flüssigkeit stattfand.

Nach der Fütterung wurden die Thiere in den stets sauber gehaltenen Käfig gebracht, die einzelnen Portionen Harn in Porzellanschalen getrennt aufgefangen. Die Darreichung einer erheblichen Wassermenge mit dem Futter hatte den Vortheil, dass oftmalige Harnauscheidung eintrat und damit ein Anhalt zur Beurtheilung des zeitlichen Verlaufs der Zuckerausscheidung gegeben war.

Zum Nachweis des Zuckers im Harn dienten je nach der Art des verwendeten Zuckers verschiedene Methoden. Die Reductionsprobe zum qualitativen Nachweis wurde stets in Form der von Worm-M $\mathfrak{u} l l \mathrm{er}^{1}$ ) angegebenen trefflichen Modification ausgeführt. Sie ist für den sicheren Nachweis kleiner Zuckermengen im nicht allzu concentrirten Hundeharn ebenso gut brauchbar, wie bei Untersuchung von Menschenharn. In einer grossen Zahl von Versuchen (etwa 160) habe ich Gelegenheit gehabt, das Ergebniss der Worm$M$ ïll er'schen Probe mit jenem der polarimetrischen Untersuchung zu vergleichen, und habe nur in 6 Fällen Nichtübereinstimmung ausserhalb der unvermeidlichen Versuchsfehler ( $\pm 0,5$ Minuten am Polarimeter = etwa 0,016 Proc. Zucker) beobachtet.

Davon betrafen 2 Versuche Fälle, wo eine Spur Reduction neben schwacher Linksdrehung vorhanden war (trotzdem rechtsdrehende Zuckerarten gereicht worden waren), ein Vorkommniss, welches sich unsehwer in der Art erklärt, dass in diesen vereinzelten Fällen die normale Linksdrehung des verdünnt entleerten Harns immer noch hinreichte, die optische Wirksamkeit der vorhandenen Zuckerspuren völlig zu verdecken.

1) Pflüger's Archiv für die ges. Physiologie. XXVII. Bd. S. 107. 
Die anderen 4 Fälle waren solche, wo die Worm-Müller'sche Probe im Stich liess, während das Polarimeter eine geringe nicht $4 \mathrm{Mi}$ nuten übersteigende Rechtsdrehung auswies. Ein solches Verhalten wurde je 1 mal nach Fitterung mit Trauben- und Milchzucker, 2 mal nach Stärkefütterung ${ }^{1)}$ beobachtet. Der Versuch sicherzustellen, ob rechtsdrehende, nicht reducirende Substanz vorlag, scheiterte an der Kleinheit der betreffenden Harnproben. Ich lasse es demnach vorläufig dahingestellt, $\cdot \mathrm{b}$ diese Vermuthung zutrifft, oder ob nur eine Behinderung der WormMüller'schen Reaction durch irgend welche sonst im Hundeharn nicht auftretende Stoffe vorlag.

Zur quantitativen Bestimmung des im Harn auftretenden Zuckers diente fast ausschliesslich die polarimetrische Bestimmung. Die Linksdrehung des normalen Hundeharns, die sich bei dieser Bestimmungsweise in störender Weise geltend macht, kommt nicht in Frage, wenn der Harn, wie dies in meinen Versuchen der Fall war, sebr verdünnt entleert wird. Immerhin muss mit der Möglichkeit gerechnet werden, dass die durch Polarisation für rechtsdrehenden Zucker gefundenen Werthe etwas zu niedrig, für linksdrehenden zu hoch gegriffen sind. Auf die Beweiskraft der unten mitzutheilenden Versuche ist dieser Umstand nicht von Einfluss.

Versuche. Von den mir vorliegenden etwa 40 einzelnen Fütterungsversuchen genügt es, im Nachstehenden nur die wichtigeren, ausschlaggebenden mitzutheilen.

\section{Traubenzucker.}

Der verwendete Traubenzucker war aschefrei, enthielt nur etwa 2 Proc. hygroskopisch festgehaltenes Wasser.

Versuch Ia. Hund A. Gut genährtes älteres Thier, $2620 \mathrm{~g}$ schwer, erhält am 5. Januar $18871 \mathrm{~h}$. Nachmittags $8 \mathrm{~g}$ Tranbenzucker in $200 \mathrm{ccm}$ Fleischbrühe.

Harn von 3 h. 20 m. $60 \mathrm{ccm}$, gelb, saner, sehr starke Reduction. Drehung im Eindecimeterrohr $+15,4^{\prime}=0,49$ Proc. $=0,29 \mathrm{~g}$ Traubenzucker. ${ }^{2}$ ) tion fehlt.

Harn von 3 h. $50 \mathrm{~m} .65 \mathrm{ccm}$, blass, amphoter reagirend. Reduc-

Versuch Ib. Derselbe Hund. Gewicht des Thieres 2600 g. Am 6. Januar 1887 um 10 b. $30 \mathrm{~m}$. Vorm. $6 \mathrm{~g}$ Traubenzucker in 220 Suppe. Harn von $11 \mathrm{~h} .30 \mathrm{~m} .30 \mathrm{ccm}$, gelb, sauer, sehr stark reducirend. Drehung $=+39,7^{\prime}=1,26$ Proc. $=0,378 \mathrm{~g}$ Traubenzucker.

Harn von 12 h. $10 \mathrm{~m}$. $55 \mathrm{ccm}$, blass, sauer, nicht reducirend.

1) Ueber die Versuche mit Stärkefütterung werde ich in einer nächsten Arbeit berichten.

2) Sämmtliche Angaben der Drehung beziehen sich auf Beobachtung im Eindecimeterrohr. Die Drehung der Dextrose ist, da es sich um sehr verdünnte Lösungen handelt, mit $+52,5^{0}$ angenommen. 
Versuch Ic. Derselbe Hund. Gewicht $2580 \mathrm{~g}$, erbält am 6. Jan. 1887 um $5 \mathrm{~h}$. Nachm. $5 \mathrm{~g}$ Traubenzuckèr in 100 Fleischbrühe.

Harn von 5 h. $35 \mathrm{~m}$. $50 \mathrm{ccm}$, gelb, sauer, nicht reducirend.

Harn von 8 h. $20 \mathrm{~m}$. $40 \mathrm{ccm}$, ebenso.

Harn vom 7. Januar früh $10 \mathrm{~h} .70 \mathrm{ccm}$; ebenso.

Versuch II a. Derselbe Hund. Durch einige Tage gut mit Fleisch gefiittert. Gewicht $2800 \mathrm{~g}$. Erhält am 11. Januar $1887 \mathrm{um} 11 \mathrm{~h} .30 \mathrm{~m}$. Vormittags $6 \mathrm{~g}$ Traubenzucker in 200 Brühe mit etwas Fleisch.

Harn von $1 \mathrm{~h} .45 \mathrm{ccm}$, blassgelb, amphoter reagirend, keine Reduction.

Harn von 4 h. $30 \mathrm{~m}$. $30 \mathrm{ccm}$, von gleichem Verhalten.

Harn vom 12. Januar früh ebenso.

Versuch II b. Derselbe Hund. Gewicht $2750 \mathrm{~g}$. Erhält am 12. Januar um 1 h. 15 m. $7 \mathrm{~g}$ Traubenzucker in 200 Brühe, daneben etwas Fleisch.

Harn von 3 h. $50 \mathrm{ccm}$, gelb, sauer, mässig stark redueirend. Drehung $=+6,3^{\prime}=0,2$ Proc. $=0,10 \mathrm{~g}$ Traubenzucker.

Harn von 3 h. 45 m. $44 \mathrm{ccm}$, blassgelb, ohne ausgesprochene Reaction gegen Lackmus; keine Reduction.

Harn von 5 h. Abends gleiches Verhalten.

V'ersuch IIc. Derselbe Hund. In der Zwischenzeit mit ungenügenden Mengen Fleisch genährt, $2610 \mathrm{~g}$ schwer. Erhält am 20. Januar 1887 um 4 h. Nachmittags $6 \mathrm{~g}$ Traubenzucker mit $200 \mathrm{~cm}$ Brihe und $100 \mathrm{~g}$ Fleisch.

Harnproben von 4 h., 7 h., 7 h. 50 m. und vom nächsten Tage sämmtlich ohne Reductionsvermögen.

Versuch III a. Junger, gut genährter Hund B. $3400 \mathrm{~g}$ schwer.

Harn vom 5. December 1886 fruh $7 \mathrm{~h} .140 \mathrm{ccm}$, hellgelb, sauer, ohne Einwirkung auf Kupferoxyd. Drehung - $3,0^{\prime}$.

Harn von 12 h. $15 \mathrm{~m}$. $35 \mathrm{ccm}$, von gleichem Verhalten. Drehung $-1,5^{\prime}$.

12 h. 45 m. 10 g. Traubenzucker in 200 Fleischbrühe.

Harn von 5 b. Nachmittags. $200 \mathrm{ccm}$, blassgelb, sauer, deutlich reducirend. Drehung $+1, l^{\prime}=$ Spur Zucker.

Harn von 6 h. 25 cem, blass, sauer; keine Reduction. Drehung $-2,5^{\prime}$.

Versuch III b. Derselbe Hund, 3300 g sehwer. Erhält am 6. December um 11 h. $30 \mathrm{~m} .8 \mathrm{~g}$ Traubenzucker in $200 \mathrm{ecm}$ Fleischbriihe. Harn von $1 \mathrm{~h}$. $35 \mathrm{ccm}$, gelb, schwach sauer, nicht reducirend. Drehung - $1,0^{\prime}$.

Harn von 1 h. $30 \mathrm{~m}$. $35 \mathrm{ccm}$, blassgelb; sonst wie voriger. Drehung $-0,5^{\prime}$.

Harn von 3 h. $45 \mathrm{~m}$. $20 \mathrm{ccm}$, wie voriger. Drehung - $0,3^{\prime}$.

Harn von $4 \mathrm{~h} .15 \mathrm{~m}$. $20 \mathrm{ccm}$, wie voriger, doch stärker sauer. Drehung - 0,6'.

Versuch IV a. Hund C. $1900 \mathrm{~g}$ schwer, mager, erhält am 28. Juli $18885 \mathrm{~h}$. Nachmittags $8 \mathrm{~g}$ Traubenzucker mit $100 \mathrm{ccm}$ Fleischbriihe, daneben $60 \mathrm{~g}$ Fleisch. 
Der Harn von 5 h., von 7 h. 15 m., von 8 h. 5 m. und vom 19. Juli früh gleicherweise ohne Reductionsvermögen.

Versuch IVb. Derselbe Hund erlält am 19. Juli um $10 \mathrm{h.} 30 \mathrm{~m}$. Vormittags $10 \mathrm{~g}$ Traubenzucker mit $150 \mathrm{ccm}$ Fleischbrühe und $60 \mathrm{~g}$ Fleisch.

Harn von 12 h. $30 \mathrm{~m}$. $55 \mathrm{ccm}$, amphoter reagirend, reducirt sehr schwach, doch unzweifelhaft $=$ Spur Traubenzucker.

Harn von 1 h. 15 m. $17 \mathrm{ccm}$, alkalisch reagirend, ohne Reductionswirkung.

Versuch V. Hund D. Grösseres, aber sehr schlecht genährtes Thier, $2450 \mathrm{~g}$ schwer. Erhält am 27 . November $188610 \mathrm{~h}$. Vorm. $10 \mathrm{~g}$ Traubenzucker in $200 \mathrm{ccm}$ Fleischbriihe. Die Harnproben von $4 \mathrm{~h}$., 5 h. 30 m., 6 h. 30 m. und 7 h. 40 m. sämmtlich ohne Reductionswirkung.

Aus den angeführten Versuchen geht hervor, dass beim Hund wie beim Menschen iiberschiissig verfïtterter Traubenzucker im Harn zum Vorschein kommt. Die Grenze der Zuckerzufuhr, bei welcher dies erfolgt, ist jedoch ziemlich verschieden.

Für den Hund A stellt sie sich laut Versuch $I b$ und $I c$ auf 5-6 g (1,9-2,3 g pro Kilo Thier), laut Versuch II a, II b und II c auf $6-7 \mathrm{~g}(2,1-2,5 \mathrm{~g}$ pro Kilo). Für den Hund $\mathrm{B}$ ergiebt sich die Grenze laut Versuch III a wenig unter $10 \mathrm{~g}$ ( $2,9 \mathrm{~g}$ pro Kilo), für den Hund $\mathrm{C}$ laut Versuch IV b ebenfalls nahe an $10 \mathrm{~g}(5,8 \mathrm{~g}$ pro Kilo); bei Hund $\mathrm{D}$ ist sie laut Versuch $\mathrm{V}$ bei $10 \mathrm{~g}(4,0 \mathrm{~g}$ pro Kilo) noch nicht erreicht.

Hierzu ist jedoch zu bemerken, dass die Berechnung pro Kilo Körpergewicht in diesem Falle einen ungenügenden Ausdruck für die Assimilationsgrenze darstellt, insofern dasselbe Thier je nach seinem Ernährungszustand, namentlich seinem Fettreichthum, ein sehr verschiedenes Gewicht besitzen kann, während doch kein Beweis dafür vorliegt, dass sich mit zunehmendem Ansatz von Fett und Fleisch u. s. w. seine Fähigkeit, Zucker zu assimiliren, steigert. Nach meinen Erfahrungen bleibt bei demselben Thier die Assimilationsgrenze fuir Zucker nahezu dieselbe, wenn sich auch der Ernährungszustand wesentlich ändert. In dieser Richtung möchte ich darauf verweisen, dass Hund B mit einem Anfangsgewicht von $3400 \mathrm{~g}$, welcher nach Fuitterung mit $10 \mathrm{~g}$, nicht aber mit $8 \mathrm{~g}$ Dextrose Zucker mit dem Harn ausschied, als er durch wiederholtes mehrtägiges Fasten und nachfolgende ungenügende Fleischzufuhr auf $2420 \mathrm{~g}$ herabgebracht war, doch nach $8 \mathrm{~g}$ Traubenzucker ebensowenig Glykosurie darbot, als vordem. Dass eine sehr weitgehende Aenderung der Ernährung die Assimilationsgrenze verschiebt, werde ich ein anderes Mal zu erörtern haben. Innerhalb physiologischer Grenzen dürfte es daher zweckmässig sein, die Assimilationsgrenze für das einzelne Versuchs- 
thier, nicht aber pro Kilo Hund festzustellen, da nur mit Berïcksichtigung dieses Umstandes ein Vergleich der Assimilirbarkeit der verschiedenen Zuckerarten mit Erfolg durchgefuihrt werden kann.

\section{Milchzucker.}

Es kam reinster Milchzucker des Handels in Verwendung.

Versuch VIa. Hund A, $2710 \mathrm{~g}$ schwer. Erhält am 13. Januar $1887 \mathrm{um} 4 \mathrm{~h}$. Nachmittags $5 \mathrm{~g}$ Milchzucker nebst $300 \mathrm{ccm}$ Fleischbrühe und etwas Fleisch.

Harn von $4 \mathrm{~h} .40 \mathrm{cem}$, concentrirt, stark gelb, amphoter reagirend, ohne Reductionsvermögen. Drehung - 4,4'.

Harn von 5 h. $30 \mathrm{~m}$. $50 \mathrm{ccm}$, blassgelb, alkalisch, mässig stark reducirend. Drehung $=+7,6^{\prime}=0,24$ Proc. $=0,12 \mathrm{~g}$ Milchzucker. ${ }^{1}$ )

Harn von $6 \mathrm{~h}$. $55 \mathrm{ccm}$, blass, neutral, schwach reducirend. Drehung: $=+2,7=0,09$ Proc. $=0,05 \mathrm{~g}$ Milchzucker.

Harn von $6 \mathrm{~h} .30 \mathrm{~m}$. $50 \mathrm{ccm}$, wie der vorige, Spur Reduction darbietend. Drehung $+0,1^{\prime}=$ Spur Zucker.

Harn von $7 \mathrm{~h}$. $40 \mathrm{~m}$. $40 \mathrm{ccm}$, wie der vorige, keine Reduction.

Versuch VIb. Derselbe Hund, $2680 \mathrm{~g}$ schwer. Erhält am 14. Januar um $4 \mathrm{~h} .15 \mathrm{~m} .4 \mathrm{~g}$ Milchzucker in $300 \mathrm{ccm}$ Brühe neben etwas Fleisch.

Harn von 4 h. $15 \mathrm{~m} .60 \mathrm{ccm}$, sehr concentrirt, gelb, alkalisch, reducirt nicht. Drehung - 7,25'.

Harn von 6 h. $30 \mathrm{~m} .135 \mathrm{ccm}$, sehr dünn, blass, amphoter reagirend, stark reducirend. Drehung $+7,5^{\prime}=0,23$ Proc. $=0,32$.

Harn von 7 h. 45 m. $45 \mathrm{ccm}$, ebenso beschaffen, aber nicht reducirend. Drehung - 0,25'.

Versuch VIc. Derselbe Hund, 2620 g schwer. Erhält am 15. Jan. $3 \mathrm{~h} .45 \mathrm{~m}$. Nachmittags $3 \mathrm{~g}$ Milchzucker mit 250 Fleischbrühe und $100 \mathrm{~g}$ Fleisch.

Harn von 5 h. $30 \mathrm{~m} .90 \mathrm{~cm}$, blass, alkalisch, schwach reducirend. Drehung $+4,1=0,13=0,12 \mathrm{~g}$ Milchzucker.

Harn von 6 h. $50 \mathrm{ccm}$, blass, neutral, nicht reducirend. Drehung $+0,3^{\prime}$.

Versuch VId. Derselbe Hund, $2590 \mathrm{~g}$ schwer. Erhält am 16. Jan. 12 h. $30 \mathrm{~m} .2 \mathrm{~g}$ Milchzucher mit $50 \mathrm{~g}$ Fleisch nebst $200 \mathrm{ccm}$ Suppe.

Harn von 12 h. $30 \mathrm{~m} .50 \mathrm{ccm}$, stark gelb, etwas alkalisch, nicht reducirend. Drehung - 3,9'.

Harn von 1 h. $20 \mathrm{~m}$. $15 \mathrm{ccm}$. blassgelb, amphoter reagirend, starke Reduction. Drehung $+4,6^{\prime}=0,15$ Proc. $=0,02 \mathrm{~g}$.

Harn von 1 h. 45 m. $20 \mathrm{ccm}$, wie der obige, mässig stark reducirrend. Drehung $+0,3^{\prime}=$ Spur Zucker.

Harn von 3 h. 30 m. $120 \mathrm{ccm}$ - zuckerfrei.

1) Vorausgesetzt, dass unveränderter Milchzucker zur Ausscheidung kommt. Es ist jedoch mit der Möglichkeit zu rechnen (s. u.), dass der ausgeschiedene Zucker Galaktose ist. Eine Entscheidung hierüber lässt sich, wo es sich um so kleine Quantitäten handelt, nicht treffen. Bei Fütterung mit noch grösseren Mengen Milchzucker werden aber die Verhältnisse möglicherweise so geändert, dass ein Rückschluss auf den vorliegenden Fall nicht statthaft erscheint. 
Versueh VI e. Derselbe Hund, $2550 \mathrm{~g}$ schwer, erhält am 17. Jan. 4 h. $1 \mathrm{~g}$ Milchzucker mit $200 \mathrm{ccm}$ Suppe und $50 \mathrm{~g}$ Fleisch.

Die Harnproben von $4 \mathrm{~h}$. an, bis zum nächsten Morgen erweisen sich sämmtlich als nicht reducirend.

Versuch VII. Hund B, $3200 \mathrm{~g}$ schwer, erhält am 7. December 188611 h. $30 \mathrm{~m} .8 \mathrm{~g}$ Milchzucker in $350 \mathrm{ccm}$ Fleischbrihe.

Harn von $11 \mathrm{~h} .30 \mathrm{~m}$. zuckerfrei.

Harn von $1 \mathrm{~h} .20 \mathrm{~m} .70 \mathrm{ccm}$, hellgelb, alkaliseh, stark reducirend. Drehung $=+4,6^{\prime}=0,15$ Proc. $=0,10 \mathrm{~g}$. Milchzucker.

Harn von 1 h. $30 \mathrm{~m}$. $45 \mathrm{ecm}$, wie obiger; schwächer reducirend. Drehuing $=+1,7^{\prime}=0,05$ Proc. $=0,02 \mathrm{~g}$.

Harn von 1 h. $55 \mathrm{~m}$. $=55 \mathrm{ccm}$, wie der vorige mässig stark reducirend. Drehung $=+2,2^{\prime}=0,07$ Proc. $=0,04 \mathrm{~g}$.

Harn von $3 \mathrm{~h} .55 \mathrm{~m}$. $=40 \mathrm{ccm}$ - zuckerfrei.

Versuch VIII. Hund D. $2500 \mathrm{~g}$ schwer, schlecht genährt, erhält am 25. November $1886 \mathrm{um} 1 \mathrm{~h} .45 \mathrm{~m} .10 \mathrm{~g}$ Milchzucker in $200 \mathrm{ccm}$ leimreicher Suppe. Harn vorher zuckerfrei.

Harn von 3 h. $45 \mathrm{~m} .70 \mathrm{ecm}$, blass, alkalisch, stark reducirend. Drehung $+11,4^{\prime}=0,36$ Proc. $=0,25 \mathrm{~g}$ Milchzucker.

Harn von 4 b. $45 \mathrm{~m}$. $30 \mathrm{ccm}$, sehr blass, neutral, ohne Reductionsvermögen.

Aus vorliegenden, namentlich aus den an Hund $A$ angestellten Versuchen geht zunächst hervor, dass Milchzncker ausserordentlich viel leichter Glykosurie veranlasst, als Traubenzucker. Die Grenze liegt bei Hund A zwischen 1-2 g Milchzucker $(=0,4-0,8 \mathrm{~g}$ pro Kilo).

Welcher Art der ausgeschiedene Zucker war, ob unveränderter Milchzucker, oder aber seine Spaltungsproducte zur Ausscheidung kamen, war durch die Untersuchung des Harns, da es sich um zu kleine Mengen handelte, nicht festzustellen.

Beim gesunden Menschen kommt nach Worm-M üller ${ }^{1}$ ) unveränderter Milchzucker im Harn zum Vorschein, nach de Jong ${ }^{2}$ ) neben diesem etwas vergährbarer Zucker. Im vorliegenden Falle musste die Wahrscheinlichkeit, dass, falls Spaltung des Milchzuckers erfolgt, Dextrose im Harn auftrete, gering erscheinen, da die Assimilirbarkeit der Dextrose nach dem fruher Angeführten eine so hohe ist. Wohl aber konnte Galaktose im Harn auftreten. Um zu sehen, welche Zuckerart zur Ausscheidung gelangt, wenn gleiche Mengen von Dextrose und Galaktose entsprechend ihrer Entstehung aus Milchzucker im Organismus zur Verfügung stehen, wurden zwei Fütterungsversuche mit invertirtem Milchzucker vorgenommen, von denen ich den einen hier anführe.

1) Pflüger's Arch. f. d. ges. Physiologie. XXXIV. Bd. S. 587.

2) Dissertation. Amsterdam 1886. Bei Stokvis gearbeitet und von diesem im Jahresbericht für Thierchemie. 1886. S. $44 \check{\text { referirt. }}$ 
Versuch IX. Hnd B. $3150 \mathrm{~g}$ sehwer. $10 \mathrm{~g}$ Milehzacker warden in Wasser gelöst, mit wenig Säure bis zur Drehungsconstanz auf dem Wasserbad erhitzt, dann nach Nentralisation der Säure mit $140 \mathrm{ccm}$ Fleischsuppe verabreicht. Zeit der Fütternng 8. December 1886 5 h. Nachmittags.

Harn von 5 h. $15 \mathrm{~m}$. $35 \mathrm{ccm}$, blass, sauer, nicht reducirend. Drehung $-0,3^{\prime}$.

Harn von 7 h. $15 \mathrm{~m} .50 \mathrm{ccm}$, blass, neutral, sehr stark reducirend, Drehung $+15,9^{\prime}(=0,315$ Proc. $=0,16 \mathrm{~g}$ Galaktose $\left.) .{ }^{1}\right)$

Harn von 7 h. $45 \mathrm{~m}$. $80 \mathrm{ccm}$, sehr blass, neutral, stark reducirend. Drehung $+12,3(=0,244$ Proc. $=0,19 \mathrm{~g}$ Galaktose $)$.

Harn von 8 h. $30 \mathrm{ccm}$, blass, schwach saner, schwach reducirend. Drehung $+6,0(=0,119$ Proc. $=0,036 \mathrm{~g})$.

Harn von 8 h. $45 \mathrm{~m}$. $60 \mathrm{ccm}$, wie der vorige. Drehung $+7,8$ $(=0,154$ Proc. $=0,092 \mathrm{~g})$.

Harn von 9. December früh. $123 \mathrm{ccm}$, blassgelb, schwach sauer, sehr schwach reducirend. Drehung $+2,1(=0,04$ Proc. $=0,05 \mathrm{~g})$.

Behufs Prüfung der Vergährbarkeit wurden die zwei zuckerreichsten Harnportionen vereinigt, mit Weinsäurelösung schwach angesäuert, aufgekocht, nach Auskïhlen nnter Baumwollverschluss mit etwas gewascheuer auf Zuckerlösung 2 mal umgezüchteter Bierhefe versetzt und so eine Nacht bei etwa $30^{\circ}$ stehen gelassen. Eine Probe Menschenharn, mit reinem Traubenzucker versetzt, wurde zum Vergleich genau so behandelt. Ansäuern und Aufkochen hatten den Zweck, Bakteriengährung, welcher die Galaktose leicht anheimfällt, hintanzmhalten. Die Untersuchung der Proben am anderen Morgen zeigte, dass in dem traubenzuckerhaltigen Controlharn der Zucker völlig vergoren war, während in dem Versuchsharn weder äussere Zeichen von Gährung vorhanden waren, noch auch bei polarimetrischer Bestimmung eine Verminderung des Zuckergehaltes nachweisbar war. Da Galaktose im Gegensatz zur Dextrose durch reine Hefe nur sehwierig in Alkoholgährung versetzt wird, so beweist dies Verhalten, dass der nach Fütterung von invertirte m Milchzucker im Harn auftretende reducirende Körper vorwiegend Galaktose sein muss.

Da Milchzucker bei der Spaltung nur etwa die Hälfte seines Gewichtes an Galaktose liefert, so müsste, falls nach Milchzuckerfütterung nur Galaktose durch die Nieren austräte, die Futterung mit Galaktose in noch kleinerer Menge Glykosurie erzielen können, als mit Milchzucker. Der Versuch spricht für die Richtigkeit dieser Schlussfolgerung.

Versuch Xa. Hund A, $2580 \mathrm{~g}$ schwer, erhält am 18. Januar 18873 b. 45 m. 1 g krystallisirte Galaktose mit $200 \mathrm{ccm}$ Rindssuppe nnd $50 \mathrm{~g}$ Fleisch.

Harn von 3 h. $30 \mathrm{~m} .60 \mathrm{ccm}$, gelb, sauer, nicht reducirend. Drehung $-4,2^{\prime}$.

Harn von 5 h. $30 \mathrm{~m} .30 \mathrm{ccm}$, blassgelb, alkalisch, deutlich reducirend. Drehung $=+4,6^{\prime}=0,095$ Proc. $=0,029 \mathrm{~g}$ Galaktose.

1) $(\alpha) \mathrm{D}=$ rund $+84^{\circ}$. 
Harn von $6 \mathrm{~h} .30 \mathrm{~m} .65 \mathrm{ccm}$, blassgelb, neutral, nicht reducirend. Drehung $-0,2^{\prime}$.

Versuch X b. Derselbe Hund, $2600 \mathrm{~g}$ schwer. Erhält am 19. Jan. 18874 h. $15 \mathrm{~m}$. Nachmittags $0,5 \mathrm{~g}$ Galaktose mit $200 \mathrm{ccm}$ Fleischbriihe und $50 \mathrm{~g}$ Fleisch.

Harn von 5 h. 50 m., sowie von 6 h. 50 m. zuckerfrei.

Vergleicht man das Ergebniss vorstehender Versuche mit jenem der Versuche VId und VI e, so sieht man, dass bei demselben Thier und unter gleichen Ernährungsverbältnissen die Futterung mit $2 \mathrm{~g}$ Milchzucker den Uebertritt annähernd der gleichen Zuckermenge in den Harn zur Folge batte, wie die Darreichung von $1 \mathrm{~g}$ Galaktose, dass ferner die Glykosurie bei Genuss von Milchzncker nach $1 \mathrm{~g}$, bei Aufnahme von Galaktose erst nach 1/2.g ausblieb. Die Assimilationsgrenze der Galaktose liegt sonach erheblich unter jener des Milchzuckers.

Die auffallend niedrige für die Auswerthung der Galaktose ermittelte Grösse veranlasste mich, am nächsten Tag durch einen Versuch mit Traubenzucker zu prüfen, ob nicht etwa die Assimilationsfähigkeit des. Thieres in der Zwischenzeit Einbusse erlitten hatte. Der Versuch (s. Vers. II c) mit Traubenzucker lehrte, dass dies nicht der Fall war.

Es ist nach diesem Verhalten anzunehmen, dass, wenn der Milchzucker im Körper gespalten wird, nur Galaktose im Harn erscheint, vorausgesetzt, dass die Menge der abgespaltenen Dextrose die für diese sichergestellte, viel höhere Assimilationsgrenze nicht übersteigt. Inwiefern und innerhalb welcher Grenzen die Spaltung des Milchzuckers im Organismus eine vollständige ist, bleibt weiterer Untersuchung vorbehalten.

Nach den Erfahrungen mit Milchzucker muss von vornherein erwartet werden, dass auch einfacher Milchgenuss bei Hunden Glykosurie veranlassen kann. In der That waren es einige überraschende Beobachtungen am Harn von mit Milch gefütterten Thieren, welche mich zuerst zu einem Vergleich der Assimilationsgrösse der verschiedenen Zuckerarten anregten. Yon den zahlreichen mir in dieser Beziehung vorliegenden Beobachtungen seien nur 3 einschlägige Versuche angefïhrt.

Versuch XI. Hund B, 3500 g schwer. Erhält am 2. December 1886 um 10 h. 45 m. 250 ccm abgerahmte Kuhmilch.

Harn vor der Fuitterung zuckerfrei.

Harn von 3 h. $45 \mathrm{~m} .60 \mathrm{ccm}$, blass, neutral, stark reducirend. Drehung $=+5,5^{\prime}$.

Harn von 4 h. $30 \mathrm{~m} . " 30 \mathrm{ccm}$, stark reducirend. 
Harn vom 3. December 1886 Morgens. $85 \mathrm{ccm}$, noch schwache Reduction. Drehung - 1,2'.

Versuch XII a. Hund E, $1850 \mathrm{~g}$ schwer. Erhält am 4. December 188612 h. 30 m. 70 cem abgerahmte Kuhmilch.

Harn vordem zuckerfrei.

Harn von 1 h. 35 m. $20 \mathrm{ccm}$, gelb, alkalisch, sehr stark reducirend. Harn von $6 \mathrm{~h}$. wieder zuckerfrei.

Versuch XIIb. Hund E, $1830 \mathrm{~g}$ schwer. Erhält am 5. December 188612 h. 45 m. 50 cem Kuhmilch.

Harn vordem zuckerfrei (Drehung - 2,0').

Harn von 6 h. Abends. $40 \mathrm{ccm}$, blass, sauer, stark reducirend. Drehung $+1,5^{\prime}$.

Später gelassener Harn wieder zuckerfrei.

\section{Rohrzucker.}

Versuch XIIIa. Hund A, $2800 \mathrm{~g}$ schwer. Erhält am 29. Jan. $18874 \mathrm{~h} .30 \mathrm{~m}$. Nachmittags $8 \mathrm{~g}$ Rohrzueker mit $100 \mathrm{~g}$ Fleisch in 300 cem Fleischbrühe.

Harn von 4 h. 30 m., 6 h., 7 h. 20 m. sowie vom nächsten Tage sämmtlich zuckerfrei.

Versu ch XIII b. Derselbe Hund, $2800 \mathrm{~g}$ schwer. Erbält am 1. Febr. 1887 um 4 h. $30 \mathrm{~m}$. Nachmittags $10 \mathrm{~g}$ Robrzucker mit $300 \mathrm{ccm}$ Fleischbrühe und $100 \mathrm{~g}$ Fleisch.

Harn von $6 \mathrm{~h} .15 \mathrm{ccm}$, blass, sauer, schwach reducirend. Drehung: $-4,0^{\prime}$.

Harn von 7 h. 30 m. $135 \mathrm{ccm}$, sehr blass, sauer, sehr schwach reducirend. Drehung - 4,2'.

Später gelassener Harn ohne Reductionsvermögen.

Versuch XIV. Hund B, $3450 \mathrm{~g}$ schwer. Erhält am 4. December 188612 h. 35 m. $10 \mathrm{~g}$ Rohrzucker mit 300 ccm Fleischbrühe.

Harn vorher ohne Reductionsvermögen.

Harn von 4 h. $240 \mathrm{ccm}$, blass, alkalisch, schwach reducirend. Drehung $-0,5^{\prime}$.

Harn von 6 h. zuckerfrei.

Versuch XV. Hund D, 2480.g schwer. Erhält am 26. November $1886 \mathrm{um} 11$ h. $30 \mathrm{~m} .10 \mathrm{~g}$ Rohrzucker mit $200 \mathrm{ccm}$ leimreicher Suppe. Harnproben von 3 h., 3 h. 45 m., 6 h. 15 m. und vom nächsten Tage sämmtlich zuckerfrei.

Wie aus Versuch XIIIb und XIV hervorgeht, tritt nach Rohrzuckerfütterung reducirende Substanz in den Harn über. Aus den Versuchen mit Hund $A$, an welchem die quantitativen Verhältnisse betreffend die verschiedenen Zuckerarten am genauesten ermittelt wurden, erhellt, dass es mehr Rohrzucker bedarf zur Erzeugung von Glykosurie, als Traubenzucker. Denn darnach hatten $6-7 \mathrm{~g}$ Dextrose hingereicht, ungefähr die gleiche Glykosurie hervorzurufen, welche erst durch $10 \mathrm{~g}$ Rohrzucker erzielbar war. 
Ueber die Natur des ausgeschiedenen reducirenden Stoffes ist aus den oben mitgetheilten Versuchen, da es sich um zu kleine Reductions- und Drehungswerthe handelt, nichts zu entnehmen. Bemerken muss ich jedoch, dass Inversion der Harnproben mit Säure an Reduction und Drehung nichts änderte, dass somit unveränderter Rohrzucker in nachweisbarer Menge nicht ausgeschieden worden war. Die Linksdrehung der Harnproben in Versuch XIIIb legt die Vermuthung nahe, dass Levulose (allein, oder neben Dextrose) vorhanden war, da eine so merkliche Linksdrehung $\left(-4^{\prime}\right)$ bei sehr verdünnten, blassen Harnen sonst nicht vorzukommen pflegt, wäbrend sie allerdings in concentrirten Harnen nichts Befremdliches hat. Nach Claude Bernard's 1) bestimmter Angabe geht beim Hunde uberschüssig verfütterter Rohrzucker zum Theil invertirt in den Harn über, der entleerte stark reducirende Harn drehte regelmässig nach links. Beim Menschen ist von Worm-M üller ${ }^{2}$ ) zwar Uebertritt von unverändertem Rohrzucker, in keinem Versuche aber von reducirendem linksdrehendem Zucker beobachtet worden. Es scheint sich hier um einen specifischen Unterschied zwischen Menseh und Hund zu handeln. Bei vor längerer Zeit zu anderen Zwecken angestellten Versuchen, bei denen ich Hunden weit iiber die Assimilationsgrenze hinausgehende Mengen Rohrzucker reichte, habe ich, wenn gleich nicht ausnahmslos, den Harn stark reducirend gefunden, und in einem Fall, wo Reductionsvermögen und optische Wirksamkeit genau verglichen wurden, war das Verhalten ein derartiges, dass die Anwesenheit von nahezu gleichen Mengen Dextrose und Levulose, somit wie im sogenannten Invertzueker, angenommen werden musste. Doch war in einzelnen Fällen auch unveränderter Rohrzucker, in einem Versuche sogar bei Abwesenbeit seiner Spaltungsproducte nachweisbar.

\section{Levulose.}

Nach den Erfabrungen von $\mathrm{K}_{\mathfrak{u}} \mathrm{lz}^{3}$ ) am Diabetiker, von WormMüller (a. a. O.) am Gesunden seheint Fruchtzucker beim Menschen nicht in den Harn ubberzugehen. Dass dies für den Organismus des Hundes nicht zutrifft, geht bereits aus den eben erwähnten Beobachtungen Bernard's und ans meinen Versuchen hervor. Immerhin scheint auch beim Hund der Uebertritt der Levulose schwieriger zu erfolgen, als jener anderer Zuckerarten (Rohrzucker wohl ausgenommen). Dadurch würde verständlicb, warum die Assimilationsgrenze

1) Leçons sur le diabète. Paris 1877. p. 270. 320.

2) Pflüger's Archiv. XXXIV. Bd. S. 586.

3) Beiträge z. Pathol. u. Therapie des Diabetes. Marburg 1874. 1. Bd. S. 130. 
für Rohrzucker höher liegt, als jene des Traubenzuckers. Die noch immer bestehende Schwierigkeit, grössere Mengen reiner Levulose zu erhalten, haben mich bisher verhindert, die Assimilationsgrenze dieser Zuckerart festzustellen. Versuche mit invertirtem Rohrzucker oder Honig sind für diesen Zweck unbrauchbar, weil man nicht von vornherein wissen kann, inwieweit die gleichzeitige Verfutterung von Dextrose auf die Verwendung der Levulose von Einfluss ist. Dass jedoch die Assimilirbarkeit der Levulose jene der Dextrose nicht allzu bedeutend übertrifft, geht aus dem nachstehenden Versuch hervor, wo eine aus Inulin durch vorsichtiges Erhitzen mit Säure, Neutralisiren und Entfärben mit Thierkohle erhaltene Fruchtzuckerlösung zur Verwendung kam.

Versuch XVI. Hund C, weleher laut Versuch IVb auf Darreichung von $10 \mathrm{~g}$ Traubenzucker geringe Glykosurie gezeigt hatte, $1900 \mathrm{~g}$ schwer, erhält am 20. Juli 1888 um 1 h. $20 \mathrm{~m} .14 \mathrm{~g}$ Levulose mit $60 \mathrm{~g}$ Fleisch und $100 \mathrm{ccm}$ Wasser. Fast sofort nach der Fütterung bricht das Thier einen Theil des Futters aus, verzehrt ihn jedoch bald wieder.

Die Harnproben, welche mit Vermeidung jeder Verunreinigung um $4 \mathrm{~h}$., 5 h., $6 \mathrm{~h} .15 \mathrm{~m}$. und $7 \mathrm{~h} .30 \mathrm{~m}$. gesammelt wurden, zeigten sämmtlich sehr starke Reduction. Sie wurden vereinigt und mit dem Polarimeter untersucht. Die Drehung war $1^{0} 13^{\prime}$ nach links. In der Nacht stellte sich Durchfall ein.

Das Ergebniss der vorgeführten Versuche lässt sich in nachstehender Weise zusammenfassen:

1. Die untersuchten Zuckerarten (Dextrose, Levulose, Galaktose, Robrzucker und Milchzucker) geben im Uebermaass genossen zur Ausscheidung von Zucker mit dem Harn Veranlassung.

2. Die Grösse, bis zu weleher die Zuckerzufuhr gesteigert werden muss, damit Uebertritt in den Harn erfolgt - die Assimilationsgrenze - ist für dasselbe Individuum und die gleiche Zuckerart zu verschiedenen Zeiten annähernd dieselbe.

3. Sie ist jedoch bei demselben Individuum für die einzelnen Zuckerarten verschieden. Am leichtesten gehen in den Harn über Galaktose und Milchzucker, viel schwieriger Dextrose, Levulose und Rohrzucker.

4. Die Menge des durch die Nieren ausgeschiedenen Zuckers steigert sich mit Erhöhung der Zuckerzufuhr.

5. Es kommt jedoch nicht die gesammte über die Assimilationsgrenze hinaus zugefïhrte Zuckermenge zur Ausscheidung, sondern nur ein kleiner Bruchtheil derselben.

Von diesen Sätzen scheint mir der an dritter Stelle angeführte im Augenblick besonders beachtenswerth. Den besten Beleg fir 
denselben liefern die an Hund A gesammelten Erfahrungen, die ich nachfolgend übersichtlich nebeneinander stelle.

\begin{tabular}{|c|c|c|c|c|}
\hline \multirow{2}{*}{$\begin{array}{l}\text { Nr. des } \\
\text { Versuchs }\end{array}$} & \multirow{2}{*}{ Zuckerart } & \multirow{2}{*}{ Gewicht des Thieres } & \multicolumn{2}{|c|}{ Assimilationsgrenze } \\
\hline & & & in $g$ & pro Kilo Thier \\
\hline $\mathrm{X}[\mathrm{II}$ & Rohrzy & & nahe an $10 \mathrm{~g}$ & $3,6 \mathrm{~g}$ \\
\hline Ib u. Ic & Traubenzucker. & $2580-2620$ & $5-6 \mathrm{~g}$ & $1,9-2,3 \mathrm{~g}$ \\
\hline II a bis II $c$ & & $2610-2800$ & $6-7 g$ & $2,1-2,5 \mathrm{~g}$ \\
\hline VI d u. VI e & Milchzucker & $2550-2590$ & $1-2 g$ & $0,4-0,8 \mathrm{~g}$ \\
\hline $\mathrm{Xau} \mathbf{X} \mathrm{b}$ & Galaktose . & $3580-2600$ & $0,5-1 \mathrm{~g}$ & $0,2-0,4 \mathrm{~g}$ \\
\hline
\end{tabular}

Dasselbe ergiebt sich, wenngleich weniger scharf, aus den Versuchen an Hund B und D. Bei $B$ trat nach Fütterung mit $8 \mathrm{~g}$ Traubenzucker (Vers. III b) keine Glykosurie ein, während die gleiche Menge Milchzucker (Vers. VII) dieselbe deutlich hervorrief. Ebenso ging bei Hund D nach Genuss von $10 \mathrm{~g}$ Traubenzucker (Vers. V) oder der gleichen Menge Rohrzucker (Vers. XV) kein Zucker in den Harn iuber, während die gleiche Menge Milchzucker (Vers. VIII) die Ausscheidung einer erheblichen Zuckermenge veranlasste.

Die Erkenntniss dieser Verschiedenheit in der Assimilirbarkeit der Zuckerarten, so merkwürdig sie im Hinblick darauf erscheint, dass gerade jene Zuckerarten, die man als Erzeugnisse des Thierkörpers anzusehen gewöhnt ist, in demselben minder gutinstige Verbrauchsbedingungen finden, ist an sich nicht geeignet, das Verständniss der betreffenden Assimilationsvorgänge zu fördern. Sie giebt uns nur den unzweideutigen Fingerzeig, dass sich diese Vorgänge nicht immer in gleicher Weise vollziehen. Ist auch für Dextrose und Levulose einerseits, für Galaktose andererseits das Ergebniss in der Regel insofern das gleiche, als sie schliesslich im Körper insgesammt in die sauerstoffreichsten Endproducte übergeführt werden, der Weg dazu muss in den beiden Fällen ein verschiedener sein. Der Grund dieser Verschiedenheit könnte, soweit es sich um Dextrose und Galaktose handelt, vielleicht darin gesucht werden, dass für Dextrose die Möglichkeit vorliegt, durch Aneinanderlagern mehrerer Molekeln unter Wasserabgabe in Glykogen überzugehen, während für Galaktose eine derartige Condensation ausgeschlossen ist. Da jedoch die Levulose sich der Dextrose gleich verhält, ohne unmittelbar in Glykogen übergehen zu können, so kann diese Vermuthung nicht aufrecht erhalten werden. Wahrscheinlicher scheint mir, dass diese Verschiedenheit auf der wesentlich abweichenden Constitution von Dextrose und Levulose einerseits und Galaktose andererseits beruht. Die beiden erstgenannten Zuckerarten sind Derivate des Mannits, lassen 
256 XIV. Hofimerster, Ueber Resorption und Assimilation der Nährstoffe.

sich, wie E. Fischer und Tafel ${ }^{1}$ ) zeigten, in einander überfübren, während die Galaktose einem anderen Alkohol, dem Dulcit, zugehört. Versuche mit anderen Abkömmlingen dieser Alkohole werden vielleicht die Richtigkeit dieser Vermuthung zu erweisen gestatten. Es liegt nahe, noch eine andere physịologische Verschiedenheit, die leichtere Vergährbarkeit der Dextrose und Levulose der Galaktose g'egenüber auf das gleiche Moment zuirückzuführen.

Die Leichtigkeit, mit welcher genossener Milchzucker zur Ausscheidung von reducirendem Zucker Veranlassung giebt, scheint auch für den Kliniker insofern von Interesse, als in derselben vielleicht der Umstand Erklärung findet, dass in der Literatur nicht wenige Fälle von Glykosurie bei Säuglingen, sowie bei auf Milchdiät gesetzten Erwachsenen verzeichnet sind. Bei den meisten dieser Beobachtungen lässt jedoch der Beweis, dass es sich wirklich um Zucker gehandelt habe, Vieles, wo nicht Alles zu wünschen übrig. Sollte sich bei dahin gerichteten sorgfältigeren Untersuchungen die Richtigkeit dieser Angaben herausstellen, so ist ihrem Verständnisse und ihrer weiteren klinischen Ausniitzung durch vorstehende Mittheilung der Weg geebnet.

1) Berichte der deutschen chem. Gesellschaft. XX. Bd. S. 2566. 